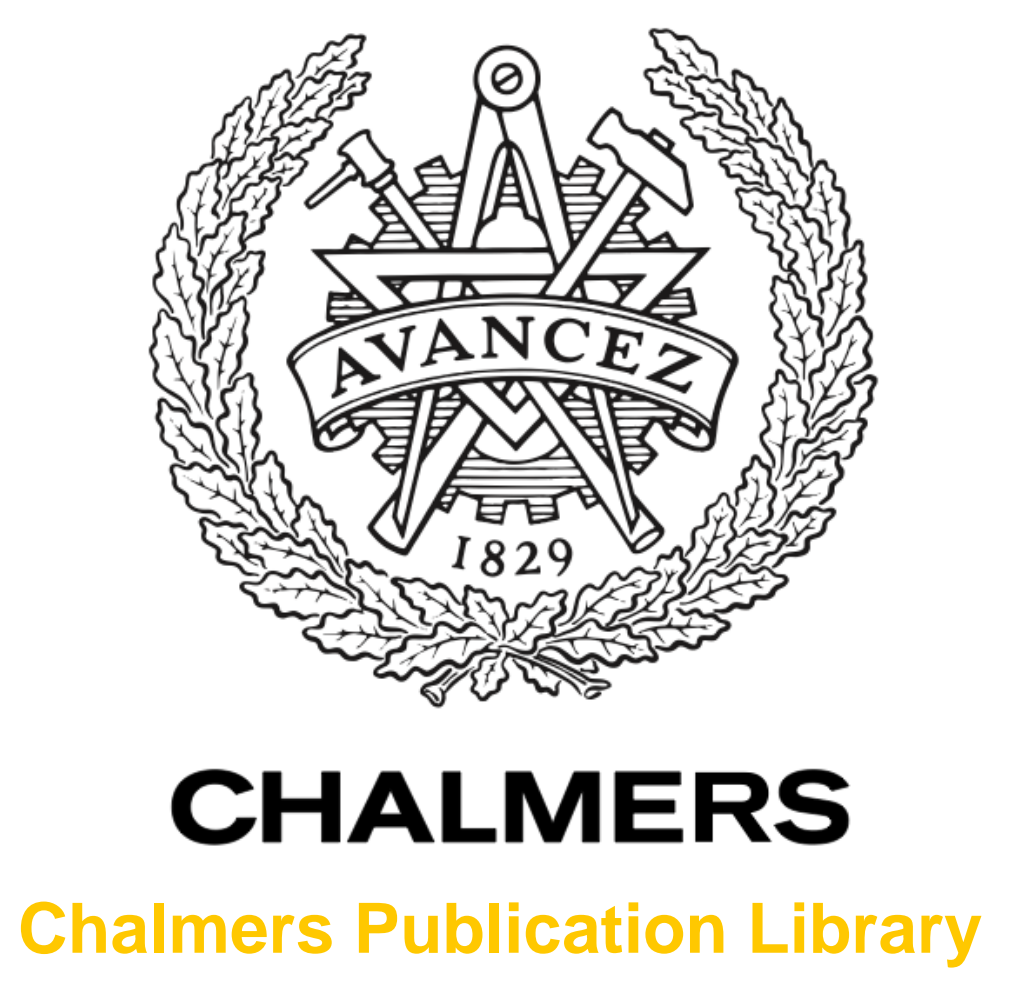

Photon upconversion facilitated molecular solar energy storage

This document has been downloaded from Chalmers Publication Library (CPL). It is the author's version of a work that was accepted for publication in:

Journal of Materials Chemistry (ISSN: 0959-9428)

Citation for the published paper:

Börjesson, K. ; Dzebo, D. ; Albinsson, B. (2013) "Photon upconversion facilitated molecular solar energy storage". Journal of Materials Chemistry, vol. 1 pp. 8521-8524.

http://dx.doi.org/10.1039/C3TA12002C

Downloaded from: http://publications.lib.chalmers.se/publication/180427

Notice: Changes introduced as a result of publishing processes such as copy-editing and formatting may not be reflected in this document. For a definitive version of this work, please refer to the published source. Please note that access to the published version might require a subscription.

Chalmers Publication Library (CPL) offers the possibility of retrieving research publications produced at Chalmers University of Technology. It covers all types of publications: articles, dissertations, licentiate theses, masters theses, conference papers, reports etc. Since 2006 it is the official tool for Chalmers official publication statistics. To ensure that Chalmers research results are disseminated as widely as possible, an Open Access Policy has been adopted.

The CPL service is administrated and maintained by Chalmers Library. 
Cite this: J. Mater. Chem. A, 2013, 1, 8521

Received 21st May 2013

Accepted 12th June 2013

DOI: $10.1039 / \mathrm{c} 3 \operatorname{ta} 12002 \mathrm{c}$

\section{Photon upconversion facilitated molecular solar energy storage $\uparrow$}

\author{
Karl Börjesson, ${ }^{\text {a Damir Dzebo, }}{ }^{\text {b }}$ Bo Albinsson ${ }^{b}$ and Kasper Moth-Poulsen*a
}

www.rsc.org/MaterialsA

Photon upconversion is used to facilitate the production of a solar fuel. This is done by collocating a triplet-triplet annihilation upconversion system in a microfluidic device with a molecular solar thermal energy storage system. Incoherent truncated white light is used to drive the reaction and the green part of the spectrum is upconverted to blue light, which in turn is absorbed by the solar fuel.

The inability to absorb sub-bandgap photons and energy relaxation to the bandgap level when absorbing high energy photons are unavoidable defects in solar energy conversion systems. The Shockley and Queisser limit sets a solar power conversion efficiency limitation on all single bandgap silicon photovoltaic devices. ${ }^{1}$ Similar limits also exist for organic photovoltaic devices as well as for molecular solar thermal systems. ${ }^{2,3}$ A generic approach to circumvent the Shockley and Queisser limit is photon upconversion. ${ }^{4,5}$ By transforming two low energy photons into one high energy photon, the solar spectrum is modified towards increased intensity in the high energy end of the spectrum. This modification is particularly relevant for energy technologies where high photon energy quanta are needed to drive energy conversion processes, such as photoinduced water splitting, ${ }^{\mathbf{6}}$ high bandgap solar cells and photoinduced chemical transformations such as molecular solar thermal systems (MOST). ${ }^{7,8}$ Photon upconversion has been around for some time, e.g. second harmonic generation, but it requires very intense coherent irradiation sources like pulsed lasers to give satisfactory quantum efficiencies. ${ }^{9}$ However, by combining fluorophores capable of performing triplet-triplet

${ }^{a}$ Chalmers University of Technology/Department of Chemical and Biological Engineering/Applied Chemistry, 41296 Göteborg, Sweden. E-mail: kasper. moth-poulsen@chalmers.se

${ }^{b}$ Chalmers University of Technology/Department of Chemical and Biological Engineering/Physical Chemistry, 41296 Göteborg, Sweden

$\dagger$ Electronic supplementary information (ESI) available: Experimental section, additional figures (negative of the photolithographic mask, experiment setup, and NMR spectra), and discussion on possible reabsorption of upconverted photons. See DOI: 10.1039/c3ta12002c annihilation (TTA) with a triplet sensitizer, photon upconversion at relatively low photon densities has been achieved in solution, on nanoparticle surfaces, and in polymer and micellar matrices. ${ }^{10-22}$ It should be noted that TTA photon upconversion as opposed to second harmonic generation does not require a coherent light source. Two recent publications have elegantly demonstrated the enhancement of solar energy production by TTA photon upconversion. ${ }^{\mathbf{2 3 2} 2}$ Laser powered TTA photon upconversion was used to facilitate $\mathrm{WO}_{3}$ catalyzed water splitting as well as for enhancing the efficiency of a hydrogenated amorphous silicon solar cell. This was carried out by co-locating a freeze-pumped cuvette containing the TTA photon upconversion material with the respective photo-reaction. Here, for the first time, we demonstrate TTA photon upconversion facilitated production of a solar fuel using non-coherent truncated white light. This is achieved by using a microfluidic device having a large illuminated area (approximately $400 \mathrm{~mm}^{2}$ ), and the ability of the TTA photon upconversion material to facilitate solar fuel production is demonstrated over several hours.

A molecular solar thermal energy storage system was used as the solar fuel. This kind of molecular system stores the solar energy in chemical bonds and has been proposed as a solution to the intermittency issues with solar energy harvesting. ${ }^{25}$ The molecular solar thermal system used in this study is a fulvalene diruthenium $\left(\mathrm{FvRu}_{2}\right)$ derivative (Fig. 1). ${ }^{7}$ Upon exposure to light, it isomerizes to a high energy photoisomer, capable of long time energy storage and on-demand energy delivery. ${ }^{26,27}$ The lowest lying absorption band of $\mathrm{FvRu}_{2}$ is centered close to $400 \mathrm{~nm}$ and tails into the visible region of the solar spectrum, whereas the high energy photoisomer does not absorb in the visible wavelength range at all, securing selective excitation of the low energy isomer. We will show that the absorption of the solar fuel is effectively extended into the 500-550 $\mathrm{nm}$ wavelength region by employing TTA photon upconversion.

The upconversion system used consists of palladium(II)octaethylporphyrin (PdOEP), which absorbs in the 500-550 nm region and 9,10-diphenylanthracene (DPA) which has a fluorescence spectrum that spectrally matches the absorption 
a

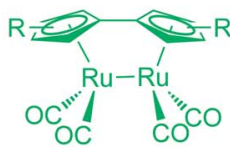
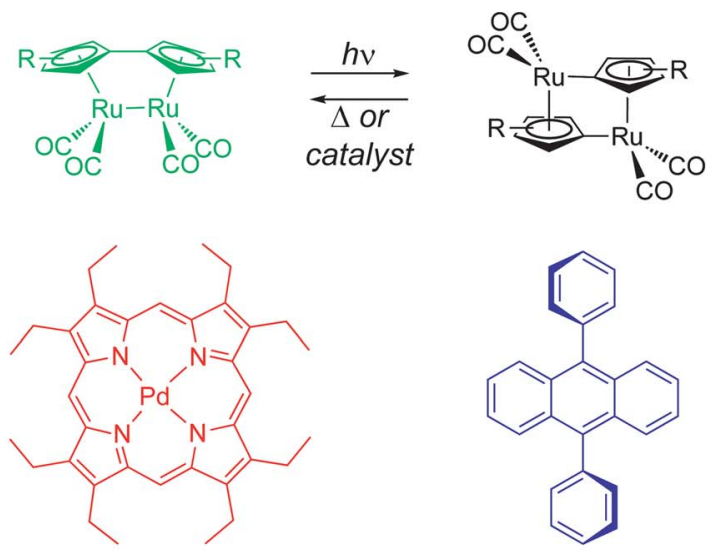

b

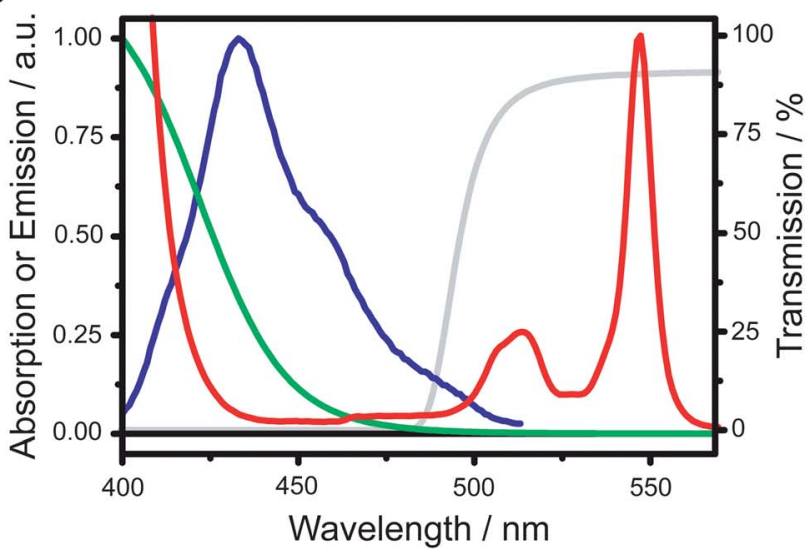

Fig. 1 (a) The fulvalene diruthenium derivative (green/black; $R=1,1$-dimethyltridecyl) used as a recyclable solar fuel and the TTA photon upconversion system based on the sensitizer PdOEP (red) and emitter DPA (blue). (b) Normalized absorption spectra of $\mathrm{FvRu}_{2}$ (green/black) and PdOEP (red), fluorescence spectrum of DPA in the presence of PdOEP (blue), and transmission spectrum of the glass filter (grey).

spectrum of the solar fuel (Fig. 1). This is a well-studied TTA photon upconversion system that has an external quantum upconversion efficiency exceeding $1 \%$ and it works as follows: ${ }^{28-30}$ after photoexcitation of the porphyrin, it quickly relaxes to the lowest lying triplet state with high quantum efficiency.

$$
{ }^{1} \mathrm{PdOEP}+h \nu_{\text {low energy }} \rightarrow{ }^{3} \mathrm{PdOEP} *
$$

While in the triplet state, the porphyrin collides with and transfers its triplet energy to the anthracene.

$$
{ }^{3} \mathrm{PdOEP} *+{ }^{1} \mathrm{DPA} \rightarrow{ }^{1} \mathrm{PdOEP}+{ }^{3} \mathrm{DPA}^{*}
$$

Two anthracenes both in their triplet state then, upon collision, undergo triplet-triplet annihilation, in which one relaxes to the ground state and the other gains energy to an excited singlet state, capable of emitting one high energy photon.

$$
\begin{gathered}
2^{3} \mathrm{DPA}^{*} \rightarrow{ }^{1} \mathrm{DPA}^{*}+{ }^{1} \mathrm{DPA} \\
{ }^{1} \mathrm{DPA}^{*} \rightarrow{ }^{1} \mathrm{DPA}+h \nu_{\text {high energy }}
\end{gathered}
$$

There is a possible shortcut to this high energy fluorescence through back sensitization of the PdOEP singlet excited state. ${ }^{31}$ Two mechanisms might be operative: non-radiative energy transfer (FRET) and radiative (trivial) energy transfer. The former is close to negligible at the PdOEP concentration used in this experiment $(0.9 \mathrm{mM}$, see ESI $\dagger)$ and the latter is minimized through the use of very short optical path lengths. Nevertheless, as can be seen in Fig. 1 the DPA fluorescence spectrum is slightly distorted in the blue edge where it overlaps with the red edge of the porphyrin Soret band. This reabsorption of the upconverted light does not severely hamper the function of the device and has therefore not been considered quantitatively in the analysis.

In order to use the upconverted photons from the PdOEP-DPA system to facilitate the energy conversion of the solar fuel a microfluidic device was constructed. The device functions as a flow reactor for continuous harvesting of solar energy, and is made up of two identical custom made microfluidic fused silica chips (Litcon). The chips contain a $60 \mu \mathrm{m}$ deep and $3.5 \mathrm{~mm}$ wide winding channel, forming a total irradiated area of $400 \mathrm{~mm}^{2}$ and an internal volume of $25 \mu \mathrm{l}$. The two glass chips are placed on top of each other with the solar fuel being pumped through the top one and the TTA photon upconversion fluid flowing through the lower chip (Fig. 2). A syringe pump was used to control the flow speed of the solar fuel (dissolved in toluene- $\mathrm{d}_{8}$ ), whereas the TTA photon upconversion system dissolved in toluene was, after careful deaeration by Argon, flowed through the chip by the aid of

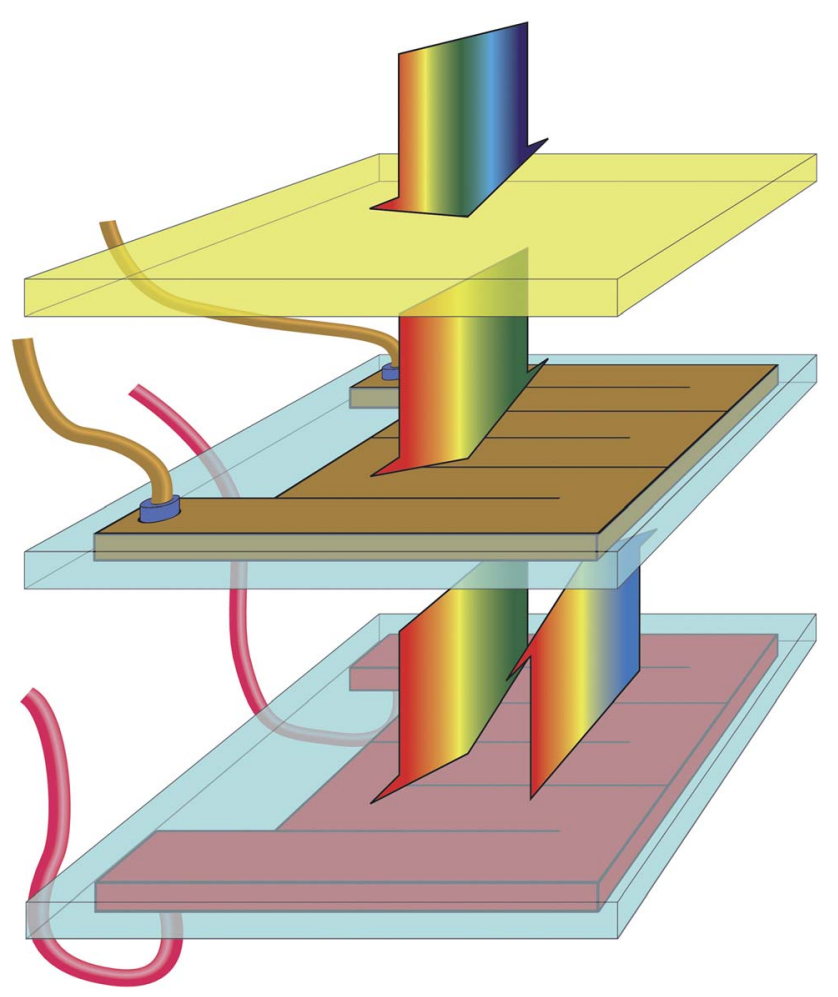

Fig. 2 A schematic picture of the microfluidic flow reactor. The white light traverses a glass filter (cut-on wavelength $=495 \mathrm{~nm}$ ) to reach the microfluidic glass chips. The first chip contains the solar fuel and in the second one the TTA photon upconversion fluid resides. 
gravity. To ensure that Argon bubbling creates a sufficiently deaerated environment for the upconversion process to occur, a control experiment in a spectrofluorometer was performed. After Argon bubbling the upconversion fluid was transferred by a similar tubing system to that used in the device to a cuvette, and the upconversion quantum yield was determined to be $0.5 \%$, which is in good agreement with the reported value of $1 \% .{ }^{28}$ At moderate light intensities the upconversion quantum yield is dependent on both light intensity and concentration, meaning that the quantum yield will not be exactly the same in the device as in this control experiment. However, the control experiment shows that the deareating and transferring methods used are sufficiently sophisticated to allow the upconversion process to occur.

In the experimental device setup, a $150 \mathrm{~W}$ metal-halide lamp was used to deliver white light having a color temperature of $4200 \mathrm{~K}$ (Osram Powerstar HQI-R). The irradiated device was immersed in a water bath $\left(30^{\circ} \mathrm{C}\right)$ to keep it at a constant temperature throughout the course of the experiment. After reaching the device, the light from the lamp first passed through a glass filter (cut-on wavelength $=495 \mathrm{~nm}$; Newport) which removed most of the high energy photons capable of direct excitation of the solar fuel. The truncated light then passed through the chip containing the solar fuel to reach the chip containing the TTA upconversion material, there being absorbed, upconverted, and reemitted to enhance the solar fuel production.

In Fig. 3 the photochemical conversion of the low energy $\mathrm{FvRu}_{2}$ isomer to the high energy photoisomer as a function of residence time in the flow reactor, in the presence and absence of TTA photon upconversion, is shown. At the very low conversions observed in this experiment the extent of reaction varies close to linearly with the residence time. The enhancement of the solar fuel production by the TTA upconversion system is $\sim 130 \%$. This enhancement is due to the light in the 500-550 $\mathrm{nm}$ wavelength range, not being absorbed by the solar fuel but instead by PdOEP, upconverted and reemitted in the 400-500 $\mathrm{nm}$ wavelength range, which is the light that the solar fuel is capable of absorbing. In the flow reactor $\mathrm{FvRu}_{2}$ is dissolved in toluene- $\mathrm{d}_{8}$, and the

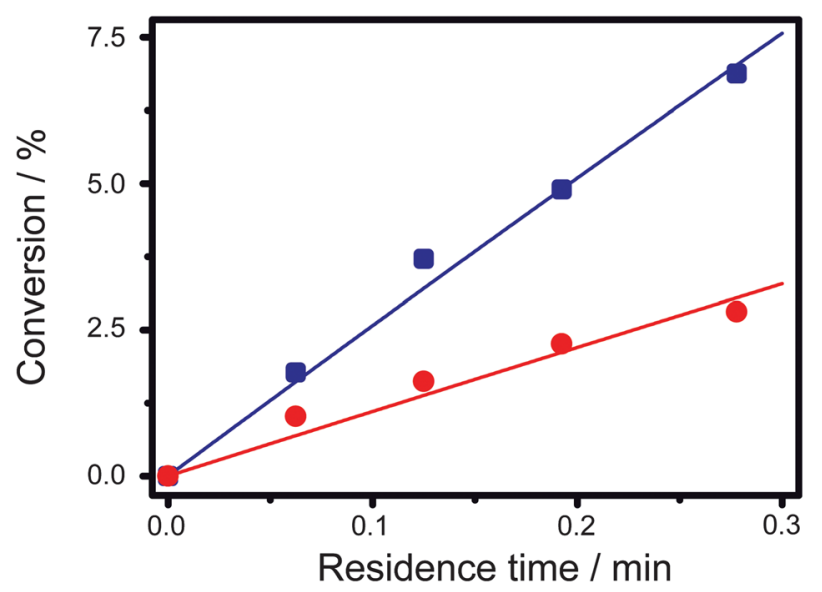

Fig. 3 Conversion efficiency (\%) of photoisomerized FvRu , in the presence (blue squares) and absence (red circles) of TTA photon upconversion, as a function of residence time in the microfluidic flow reactor. photochemical conversion is monitored by NMR without further sample preparation from the fluid exiting the flow reactor. This requires an accumulated volume of $0.5 \mathrm{ml}$. Thus, each data point in Fig. 3, showing the photochemical conversion as a function of residence time in the flow reactor, is the average result of 20 reactor volumes (which equals to 1.4-6.1 $\mathrm{h}$ depending on the flow speed). The data points are taken in random order and no fouling of the channels could be observed by the naked eye after the complete dataset was acquired, showing, at least on a lab scale, the high stability of the system. A closer examination of Fig. 3 reveals that the data points having a longer residence time are slightly biased to lower photochemical conversion than those having a shorter residence time. This is true both in the presence and absence of TTA photon upconversion, indicating that this phenomenon is not related to the upconversion as such but rather to the stability of the photoisomer. The photoisomer is sensitive to molecular oxygen, and the longer residence time requires a longer storage time from photoconversion to analysis, during which some oxygen might have reacted with the photoproduct. From an application point of view this tendency of degradation by molecular oxygen should not be a major problem since this kind of solar fuel is intended to work in a closed cycle with no oxygen contact.

\section{Conclusions}

In summary, we have shown for the first time that non-coherent light can be used to drive a TTA photon upconversion process which in turn is used to facilitate a solar energy harvesting reaction. This has been achieved by locating upconversion materials and a molecular solar thermal system on top of each other in a microfluidic device, working as a flow reactor, having an irradiated surface area of $400 \mathrm{~mm}^{2}$. The setup is stable in the sense that the solar fuel production scales with the residence time of the reactor, and that no fouling in the microfluidic channels, even after $50 \mathrm{~h}$ of run time, could be observed. When considering practical applications, one limiting factor in the used $\mathrm{FvRu}_{2}$ system is the quantum yield of photochemical conversion of $0.2 \%$ in toluene. For efficient and practical applications, new MOST systems with near unity quantum yield are preferred. ${ }^{3}$ We believe that employing TTA photon upconversion will become a general, straightforward and economically feasible method to supersede the Shockley and Queisser limit of solar light harvesting devices in the course of time. In this regard, the development of more efficient TTA-UC systems operating under natural solar irradiation conditions is highly desirable.

\section{Notes and references}

1 W. Shockley and H. J. Queisser, J. Appl. Phys., 1961, 32, 510519.

2 M. J. Y. Tayebjee, L. C. Hirst, N. J. Ekins-Daukes and T. W. Schmidt, J. Appl. Phys., 2010, 108, 124506-124512.

3 K. Börjesson, A. Lennartson and K. Moth-Poulsen, ACS Sustainable Chem. Eng., 2013, 1, 585-590. 
4 N. J. Ekins-Daukes and T. W. Schmidt, Appl. Phys. Lett., 2008, 93, 063507.

5 A. C. Atre and J. A. Dionne, J. Appl. Phys., 2011, 110, 034505.

6 Y. Y. Cheng, T. Khoury, R. Clady, M. J. Y. Tayebjee, N. J. Ekins-Daukes, M. J. Crossley and T. W. Schmidt, Phys. Chem. Chem. Phys., 2010, 12, 66-71.

7 K. Moth-Poulsen, D. Coso, K. Börjesson, N. Vinokurov, S. K. Meier, A. Majumdar, K. P. C. Vollhardt and R. A. Segalman, Energy Environ. Sci., 2012, 5, 8534-8537.

8 A. M. Kolpak and J. C. Grossman, J. Chem. Phys., 2013, 138, 3156-3162.

9 G. S. He, L. S. Tan, Q. Zheng and P. N. Prasad, Chem. Rev., 2008, 108, 1245-1330.

10 T. N. Singh-Rachford and F. N. Castellano, Coord. Chem. Rev., 2010, 254, 2560-2573.

11 Y. C. Simon and C. Weder, J. Mater. Chem., 2012, 22, 2081720830.

12 J. Z. Zhao, S. M. Ji and H. M. Guo, RSC Adv., 2011, 1, 937-950. 13 S. Baluschev, V. Yakutkin, G. Wegner, T. Miteva, G. Nelles, A. Yasuda, S. Chernov, S. Aleshchenkov and A. Cheprakov, Appl. Phys. Lett., 2007, 90, 181103.

14 A. Turshatov, D. Busko, S. Baluschev, T. Miteva and K. Landfester, New J. Phys., 2011, 13, 083035.

15 T. N. Singh-Rachford, J. Lott, C. Weder and F. N. Castellano, J. Am. Chem. Soc., 2009, 131, 12007-12014.

16 R. R. Islangulov, J. Lott, C. Weder and F. N. Castellano, J. Am. Chem. Soc., 2007, 129, 12652-12653.

17 A. Monguzzi, M. Frigoli, C. Larpent, R. Tubino and F. Meinardi, Adv. Funct. Mater., 2012, 22, 139-143.

18 P. B. Merkel and J. P. Dinnocenzo, J. Lumin., 2009, 129, 303306.

19 J. S. Lissau, J. M. Gardner and A. Morandeira, J. Phys. Chem. $C, 2011,115,23226-23232$.
20 Y. Y. Cheng, B. Fuckel, T. Khoury, R. Clady, N. J. EkinsDaukes, M. J. Crossley and T. W. Schmidt, J. Phys. Chem. A, 2011, 115, 1047-1053.

21 S. M. Ji, W. H. Wu, W. T. Wu, H. M. Guo and J. Z. Zhao, Angew. Chem., Int. Ed., 2011, 50, 1626-1629.

22 J. H. Kim, F. Deng and F. N. Castellano, Chem. Mater., 2012, 24, 2250-2252.

23 R. S. Khnayzer, J. Blumhoff, J. A. Harrington, A. Haefele, F. Deng and F. N. Castellano, Chem. Commun., 2012, 48, 209-211.

24 Y. Y. Cheng, B. Fuckel, R. W. MacQueen, T. Khoury, R. Clady, T. F. Schulze, N. J. Ekins-Daukes, M. J. Crossley, B. Stannowski, K. Lips and T. W. Schmidt, Energy Environ. Sci., 2012, 5, 6953-6959.

25 T. J. Kucharski, Y. C. Tian, S. Akbulatov and R. Boulatov, Energy Environ. Sci., 2011, 4, 4449-4472.

26 Y. Kanai, V. Srinivasan, S. K. Meier, K. P. C. Vollhardt and J. C. Grossman, Angew. Chem., Int. Ed., 2010, 49, 89268929.

27 M. R. Harpham, S. C. Nguyen, Z. Hou, J. C. Grossman, C. B. Harris, M. W. Mara, A. B. Stickrath, Y. Kanai, A. M. Kolpak, D. Lee, D. Liu, J. P. Lomont, K. MothPoulsen, N. Vinokurov, L. X. Chen and K. P. C. Vollhardt, Angew. Chem., Int. Ed., 2012, 51, 7692-7696.

28 S. Baluschev, T. Miteva, V. Yakutkin, G. Nelles, A. Yasuda and G. Wegner, Phys. Rev. Lett., 2006, 97.

29 A. Haefele, J. Blumhoff, R. S. Khnayzer and F. N. Castellano, J. Phys. Chem. Lett., 2012, 3, 299-303.

30 A. Monguzzi, J. Mezyk, F. Scotognella, R. Tubino and F. Meinardi, Phys. Rev. B: Condens. Matter Mater. Phys., 2008, 78, 195112.

31 P. E. Keivanidis, S. Baluschev, G. Lieser and G. Wegner, ChemPhysChem, 2009, 10, 2316-2326. 